\title{
Diagnostisches und therapeutisches Vorgehen bei Adipositas im Kindes- und Jugendalter
}

\author{
Caroline Knop, Thomas Reinehr
}

\section{Übersicht}

Einleitung

\section{5}

Definition der Adipositas im

Kindes- und Jugendalter

Ursachen der Adipositas im

Kindes- und Jugendalter

Diagnostisches Vorgehen bei

Adipositas im Kindes- und

Jugendalter

Prävention der Adipositas

im Kindes- und Jugendalter

117
Lebensstilintervention bei Adipositas

im Kindes- und Jugendalter

\begin{tabular}{|l|}
\hline 121 \\
\hline 123 \\
\hline 126 \\
\hline 127 \\
\hline 128 \\
\hline 129 \\
\hline
\end{tabular}

Gesprächsbeispiele

Weiterführende Behandlung

Medikamentöse Therapie

Bariatrische Chirurgie

Therapie der Folgeerkrankungen

Audio-Podcast online!

Sie finden den Audio-Podcast unter

www.thieme-connect.de/products

bei Ihrer Pädiatrie up2date

\section{Einleitung}

Übergewicht ist auch im Kindes- und Jugendalter keineswegs nur ein kosmetisches Problem, sondern stellt eine bedeutende Volkskrankheit dar [1]. Zum einen werden aus übergewichtigen Kindern meist auch übergewichtige Erwachsene, wobei das Risiko mit Alter und Ausmaß des Übergewichts zunimmt. In vielen Untersuchungen ist eindeutig ein Zusammenhang von Übergewicht und Diabetes mellitus Typ 2, Herz-Kreislauf-Erkrankungen, Fettstoffwechselstörungen sowie Arthrose belegt (Abb.1). Dabei sind die Mortalität und Morbidität umso höher, je früher die Adipositas im Kindesalter beginnt [2]. Auch das Krebsrisiko ist bei Übergewichtigen deutlich erhöht (Mammakarzinom, Endometriumkarzinom, Darmkrebs, Prostatakrebs).

\section{Definition der Adipositas im Kindes- und Jugendalter}

Die Adipositas ist durch einen erhöhten Körperfettanteil definiert. Die Dual-Röntgen-Absorptiometrie stellt die Methode der Wahl dar, um den Körperfettanteil zu messen. Diese ist jedoch sehr aufwendig und invasiv, so dass sie für die Praxis ungeeignet ist. Übergewicht und Adipositas werden daher im Kindes- und Jugendalter anhand alters- und geschlechtsbezogener

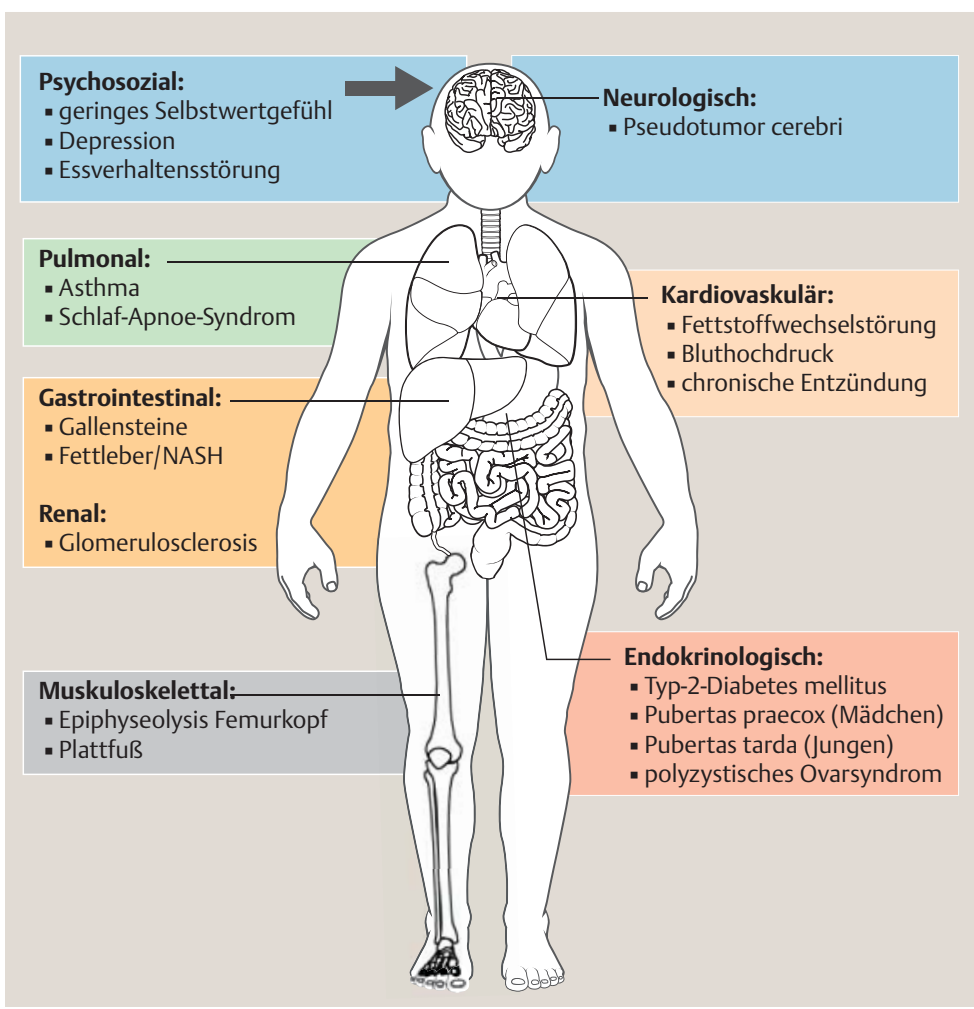

Abb. 1 Folgeerkrankungen der Adipositas im Kindes- und Jugendalter. 


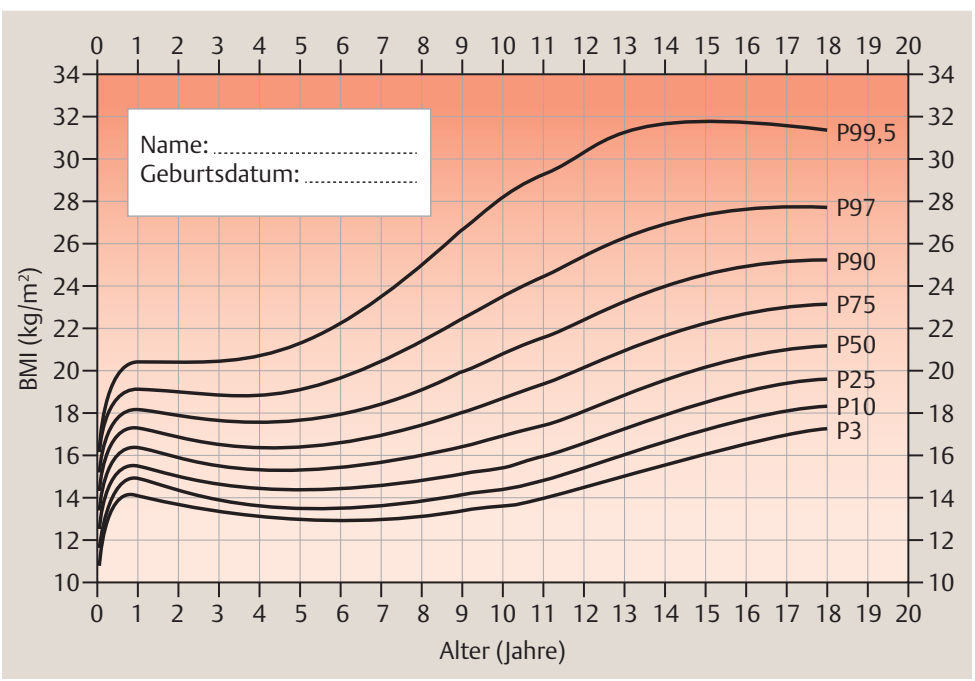

Abb. 2 BMI-Perzentilen für deutsche Mädchen.

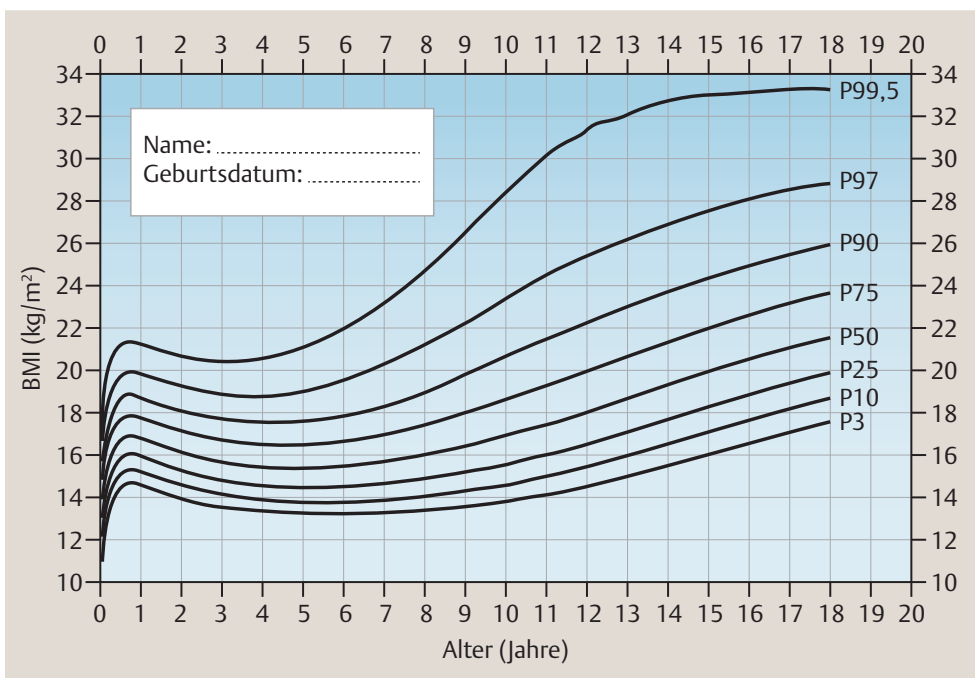

Abb. 3 BMI-Perzentilen für deutsche Jungen.

Perzentilen für den Body Mass Index (BMI) definiert (Abb.2 und Abb.3).

In Deutschland sind 8,7\% der Kinder und Jugendlichen übergewichtig und weitere 6,3\% adipös [4]. Dies ist etwa doppelt so viel wie noch vor 25 Jahren. Die Prävalenz von Übergewicht und Adipositas ist in den Industriestaaten und damit auch in Deutschland jedoch in den letzten 10 Jahren nicht mehr weiter steigend [5]. Kinder aus der unteren sozialen Schicht und Kinder aus Familien mit Migrationshintergrund haben ein höheres Risiko übergewichtig zu werden [4].
Definition von Übergewicht und

Adipositas anhand der BMI-Werte [3]

- Übergewicht: 90. bis 97. Perzentile

- Adipositas: 97. bis 99,5. Perzentile

- extreme Adipositas: >99,5. Perzentile

\section{Ursachen der Adipositas im Kindes- und Jugendalter}

Genetische Faktoren, menschliches Verhalten, Umweltund Lebensbedingungen sind multifaktoriell an der Entstehung der Adipositas beteiligt.

Umgebungsfaktoren. Für den dramatischen Anstieg der Prävalenz der Adipositas sind vor allem die deutlichen Veränderungen von Umgebungsfaktoren verantwortlich. Fehlende Bewegungs- und Spielbereiche beeinflussen das Bewegungsverhalten der Kinder ungünstig.

Verhalten. Der Grad der körperlichen Aktivität von Kindern wird zudem maßgeblich durch den Grad der körperlichen Aktivität der Eltern beeinflusst. Der zunehmende Medien- und Fernsehkonsum hat in den letzten Jahren zu einem deutlichen Rückgang der täglichen körperlichen Aktivität auch bei Kindern geführt.

Ernährung. Die Prävalenz und das Ausmaß der Adipositas korrelieren mit der Menge des konsumierten Fetts und dem Anteil gesüßter Getränke. Viele Süßigkeiten und Fast-Food-Gerichte stellen fettreiche Nahrungsmittel dar. Darüber hinaus nimmt der tägliche Verzehr von beiläufig konsumierten Lebensmitteln („Snacks“) mit hoher Energiedichte zu.

Psychische Faktoren. Essen wird auch eingesetzt, um Stress und Frust abzubauen, Trauer und Ängste kurzfristig zu betäuben und Langeweile zu überbrücken. Dieses emotionsinduzierte Essverhalten führt durch eine Entkopplung der Nahrungsaufnahme vom Hunger häufig zur Aufnahme kalorienreicher Nahrungsmittel. Familiäre Bedingungen wie Scheidung, elterliche Berufstätigkeit oder Vernachlässigung können daher ebenfalls eine wichtige Rolle bei der Genese einer Adipositas im Kindesalter spielen.

Somatische Erkrankungen. Sie sind als Ursachen des Übergewichts mit einer Häufigkeit von weniger als $1 \%$ sehr selten [6]. Neben einer genetischen Veranlagung kommen Erkrankungen mit Veränderungen der Hirnregionen, die unser Sättigungsverhalten bestimmen, 
wie das Prader-Willi-Syndrom, ein Kraniopharyngeom oder andere Tumoren im Bereich des Hypothalamus, neben endokrinologischen Erkrankungen als somatische Ursachen in Betracht.

Medikamente. Adipositas im Kindes- und Jugendalter kann auch durch Medikamente ausgelöst oder verstärkt werden. Neben systemisch verabreichten Glukokortikoiden führen auch Psychopharmaka und Antiepileptika wie Valproat, Vigabactrin, Gabapentin und Carbamazepin zu einer deutlichen Gewichtszunahme, während beispielsweise das Antiepileptikum Topiramat mit einer Gewichtsreduktion einhergeht.

\section{Chronische Erkrankungen und Behinderungen. Dane-} ben können alle chronischen Erkrankungen und Behinderungen, die mit einer Bewegungseinschränkung und oder sozialen Isolation einhergehen, zu einer Adipositas führen [7]. So sind beispielsweise Kinder mit Spina bifida, juveniler rheumatoider Arthritis, aber auch Kinder mit geistiger Behinderung deutlich häufiger übergewichtig als ihre gesunden Altersgenossen.

\section{Diagnostisches Vorgehen bei Adipositas im Kindes- und Jugendalter}

Die medizinische Diagnostik der Adipositas im Kindesund Jugendalter umfasst 3 Schritte:

1. Bestimmung des Ausmaßes des Übergewichts

2. Ausschluss von Grunderkrankungen des Übergewichts

3. Erfassung von Folgeerkrankungen durch das Übergewicht

\section{Ausmaß des Übergewichts}

Das Ausmaß des Übergewichts wird durch den Body Mass Index erfasst (s.o.). Ferner können Hautfaltendickemessungen zur Abschätzung der Körperfettmasse verwendet werden. Diese Messmethode ist jedoch sehr untersucherabhängig.

Merke: Die Messung des Bauchumfangs in Relation zur Körperhöhe (Waist-to-Height-Ratio) ist hilfreich, um Kinder mit erhöhtem Risiko für kardiovaskuläre Erkrankungen zu identifizieren [8]. Werte $>0,5$ zeigen ein erhöhtes Risiko an.

\section{Grunderkrankungen}

Adipöse Kinder sind in der Regel größer als ihre Altersgenossen. Daher dienen insbesondere die Körpergröße und die Wachstumsgeschwindigkeit dem Ausschluss von Grunderkrankungen. Bei Kleinwuchs oder einer verminderten Wachstumsgeschwindigkeit sollten eine Autoimmunhypothyreose (Schilddrüsenhormone), ein Cushing-Syndrom (Dexamethasonhemmtest), Pseudohypoparathyreoidismus (Serum-Kalzium) und ein Wachstumshormonmangel (entsprechende Stimulationstests) ausgeschlossen werden.

Cave: Bei der Beurteilung von Hormonwerten sind bei adipösen Kindern einige Besonderheiten zu berücksichtigen. Adipöse Kinder zeigen häufig grenzwertig erhöhte TSH-Werte (<10 mU/ml) bei hochnormalen fT3 - und fT4-Werten, ohne dass eine Hypothyreose vorliegt [9]. Eine medikamentöse Therapie ist nicht erforderlich.

Am ehesten handelt es sich dabei um einen Kompensationsmechanismus des Körpers zur Erhöhung des Grundumsatzes bei Übergewicht. Ferner sind die Kortisolspiegel im Urin und Blut adipöser Kinder mit metabolischem Syndrom (s.u.) erhöht [10]. Ein Cushing-Syndrom kann nur mittels Dexamethasonhemmtest sicher ausgeschlossen werden.

Leitsymptome syndromaler Erkrankungen sind Kleinwuchs, geistige Retardierung und Dysmorphiestigmata. Am häufigsten unter den insgesamt seltenen Syndromen findet man das Prader-Willi-Syndrom und das Bardet-Biedl-Syndrom. Diese und weitere syndromale Erkrankungen sind in Tab. 1 charakterisiert.

Unter den genetischen Erkrankungen spielt der autosomal dominant vererbte Melanocortin-4-Rezeptor(MC4R-)Defekt die wichtigste Rolle. Dieser ist bei 5\% der adipösen Kinder zu finden [11]. Aufgrund der fehlenden therapeutischen Konsequenz ist von einem generellen Screening bei adipösen Kindern eher abzusehen.

\section{Folgeerkrankungen}

Im dritten Schritt sind die Folgeerkrankungen der Adipositas zu erfassen. Kardiovaskuläre Risikofaktoren sind häufig Folgen der Adipositas (Abb.4) und können bereits frühzeitig zu Gefäßveränderungen führen, messbar an der Intima-Media-Dicke der A. carotis communis im Ultraschall [12]. 


\section{Tabelle 1}

Einteilung syndromaler Erkrankungen.

\begin{tabular}{|c|c|}
\hline Syndrom & Charakteristika \\
\hline \multicolumn{2}{|c|}{ Syndromale Erkrankungen mit Kleinwuchs } \\
\hline Prader-Willi-Syndrom & $\begin{array}{l}\text { - Imprinting-Erkrankung auf Chromosom 15q11-q13 } \\
\text { - „Floppy Infant“: muskuläre Hypotonie als Neugeborenes und Säugling } \\
\text { - Trinkschwäche als Säugling, aber Hyperphagie im Kleinkindalter } \\
\text { - Hodenhochstand } \\
\text { - z.T. Hypogonadismus }\end{array}$ \\
\hline Cohen-Syndrom & $\begin{array}{l}\text { - autosomal rezessiv: zu } 30 \% \text { genetisch nachweisbar } \\
\text { - schmale Hände und Füße } \\
\text { - lange Finger und Zehen } \\
\text { - offener Mund, vorstehende Schneidezähne } \\
\text { muskuläre Hypotonie } \\
\text { - Mikrozephalie } \\
\text { - mentale Retardierung } \\
\text { z.T. Optikusatrophie }\end{array}$ \\
\hline Alström-Syndrom & $\begin{array}{l}\text { - autosomal rezessiv: zu } 50 \% \text { genetisch nachweisbar } \\
\text { - charakteristisches Gesicht (tiefliegende Augen, rundes Gesicht, fleischige Ohren, vorzeitige Stirnglatze, dünne Haare) } \\
\text { - Lichtscheue und Nystagmus, Erblinden in der Regel ab einem Alter von } 12 \text { Jahren } \\
\text { - sensorineurale Schwerhörigkeit } \\
\text { z. T. Hypothyreose, z. T. hypogonadotroper Hypogonadismus } \\
\text { - kaum entwicklungsretardiert } \\
\text { - dilatative Kardiomyopathie } \\
\text { - Typ-2-Diabetes mellitus und metabolisches Syndrom häufig }\end{array}$ \\
\hline Bardet-Biedl-Syndrom & $\begin{array}{l}\text { - autosomal rezessiv vererbt, } 15 \text { verschiedene Gene betroffen } \\
\text { - postaxiale Hexadaktylie } \\
\text { - Retinitis pigmentosa (Makuladegeneration) } \\
\text { - frühes Nierenversagen (Zystenniere) } \\
\text { - früher Diabetes mellitus } 2 \\
\text { - chronische Niereninsuffizienz bei polyzystischer Nierenerkrankung }\end{array}$ \\
\hline \multicolumn{2}{|c|}{ Syndromale Erkrankungen ohne Kleinwuchs } \\
\hline Fragiles X-Syndrom & $\begin{array}{l}\text { - Makrozephalie } \\
\text { - große Hoden } \\
\text { - große Ohren } \\
\text { - Intelligenzminderung }\end{array}$ \\
\hline Sotos-Syndrom & $\begin{array}{l}\text { zu 2/3 Veränderungen im NSD1-Gen Chromosom } 5 \text { (5q35) } \\
\text { - Makrosomie bei Geburt } \\
\text { - Makrozephalie } \\
\text { - längliches Gesicht } \\
\text { - Knochenalter akzeleriert } \\
\text { - große Hände und Füße } \\
\text { - "Geheimratsecken“ } \\
\text { - Balkonstirn } \\
\text { - z.T. mentale Retardierung }\end{array}$ \\
\hline
\end{tabular}




\section{Metabolisches Syndrom}

Merke: Treten zentrale (= abdominelle) Adipositas, Fettstoffwechselstörung (Hypertriglyzeridämie und Hypo-HDL-Cholesterinämie), arterielle Hypertonie und Glukosestoffwechselstörung gemeinsam auf, so spricht man vom metabolischen Syndrom.

Dieses Clustering von Risikofaktoren, deren gemeinsame pathogenetische Grundlage die Insulinresistenz darstellt, geht mit einem deutlich erhöhten Risiko für kardiovaskuläre Erkrankungen einher. Vor allem adipöse Kinder mit einer arteriellen Hypertonie, Dyslipidämie und Glukosestoffwechselstörung zeigen eine Verdickung der Intima und Media der A. carotis communis [14].

Die Insulinresistenz wird vor allem durch Bewegungsmangel und intraabdominelles Fettgewebe verschlechtert. Mittlerweile ist gesichert, dass Fettgewebe nicht nur ein großes Speicherorgan für Energie, sondern auch ein sehr aktives endokrines Organ darstellt. Die meisten im Fettgewebe produzierten Adipozytokine (z.B. Leptin, Retinol-bindendes Protein 4, Visfatin, Resistin) führen zu einer Verschlechterung der Insulinwirkung. Interessanterweise ist Adiponectin, das einzige die Insulinresistenz verbessernde Adipozytokin, bei der zentralen Adipositas erniedrigt, wobei die Ursache noch nicht geklärt ist.

\section{- Diabetes mellitus Typ 2}

Dieser ist charakterisiert durch die Kombination von Insulinresistenz und Insulinsekretionsdefizit. Eine genetische Prädisposition liegt meist vor, so dass häufig Eltern oder Großeltern ebenfalls betroffen sind. Die Häufigkeit des Typ-2-Diabetes mellitus ist in Deutschland parallel zur Adipositas zunehmend. Zurzeit sind etwa 1000 Jugendliche mit Typ-2-Diabetes mellitus in Deutschland registriert [15]. Aufgrund von Screeninguntersuchungen haben $10 \%$ der adipösen Jugendlichen eine pathologische Glukosetoleranz (Vorstufe des Diabetes mellitus Typ 2) und 0,5-1\% einen Diabetes mellitus Typ 2 [15].

Cave: Vermutlich ist nur jeder 3. Jugendliche mit Typ-2-Diabetes mellitus bekannt [15]. Da der Diabetes mellitus Typ 2 asymptomatisch ist, sind Screeninguntersuchungen für adipöse Kinder mit Risikofaktoren (s.u.) sinnvoll.

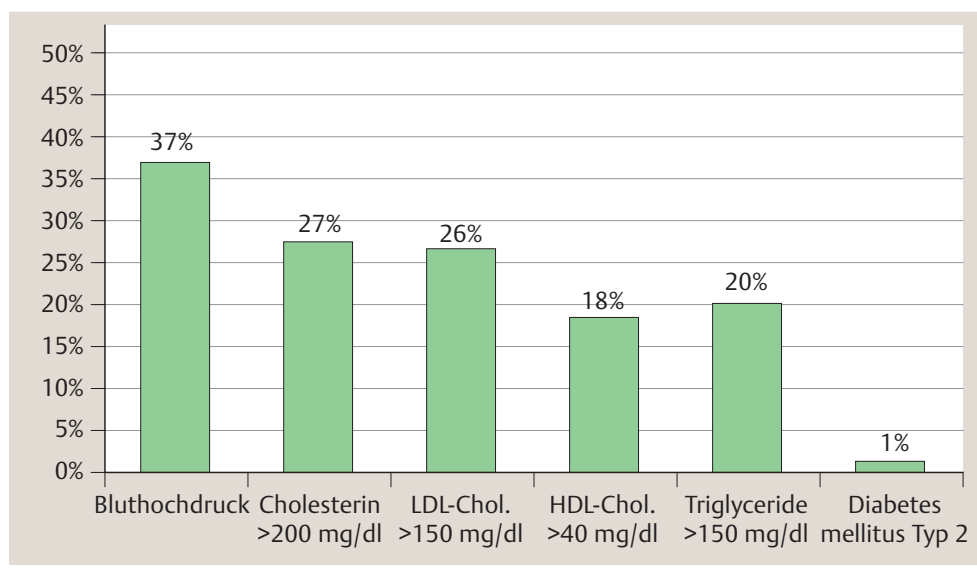

Abb. 4 Häufigkeit kardiovaskulärer Risikofaktoren bei übergewichtigen deutschen Kindern [13].

Untersuchungen bei adipösen Kindern zum Ausschluss von Folgeerkrankungen nach den Leitlinien der Arbeitsgemeinschaft Adipositas im Kindes- und Jugendalter (AGA) [3]

- Blutdruckmessung

- Eltern mit Typ-2-Diabetes

- Nüchternbestimmung von Triglyzeriden, HDL- und LDL- Cholesterin sowie Transaminasen - extrem adipöse Kinder

- Hirsutismus oder Zyklusstörungen: Vorstellung beim pädiatri-

- oraler Glukosetoleranztest ab Beginn der Pubertät zur Erfassung einer pathologischen Glukosetoleranz oder Diabetes mellitus Typ 2 bei folgenden Risikofaktoren: schen Endokrinologen zum Ausschluss eines polyzystischen Ovarsyndroms

- bei Tagesmüdigkeit oder Schnarchen: Schlaflaboruntersuchung

Die Diagnose eines asymptomatischen Diabetes mellitus Typ 2 nur aufgrund von Laborwerten muss durch 2 Tests an unabhängigen Tagen erfolgen, da der oGTT eine relativ geringe Reproduzierbarkeit hat.

\section{- Sonstige Folgeerkrankungen}

Orthopädische Probleme (z.B. Knicksenkfuß, Genu valgum), Infektionen in den Hautfalten und endokrinologische Erkrankungen (z. B. relativer Großwuchs, Pubertas praecox und polyzystisches Ovarsyndrom bei jugendlichen Mädchen [PCOS]) sind bei adipösen Kindern und Jugendlichen relativ häufig.

Ein PCOS ist gekennzeichnet durch einen Hirsutismus, Hyperandrogenämie und Zyklusstörungen.

Eine Epiphyseolysis capitis (Hüftkopfnekrose) tritt in einer akuten Form mit heftigen Schmerzen und Bewegungseinschränkungen, häufiger aber auch als chronische Form mit Hüft- und Kniegelenkbeschwerden auf. 
Bei einem Teil der übergewichtigen Kinder (v.a. Jungen) ist auch schon eine Fettleber (nichtalkoholische Steatohepatitis =NAFLD) nachweisbar. Typischerweise sind dabei die Serumspiegel der Alanintransferase (ALT) höher als die der Aspartattransaminase (AST). Die Prognose ist meist gut, selten kann die NAFLD jedoch auch in eine Leberzirrhose münden.

Gallensteine treten vor allem bei einer raschen Gewichtsreduktion auf.

Ein Schlaf-Apnoe-Syndrom als Folge einer extremen Adipositas kann durch die Tagesmüdigkeit und Antriebsarmut die Adipositas noch weiter verstärken.

Bei Adipositas führen die Stammfettmassen auch zu einer mechanisch induzierten respiratorischen Dysfunktion (AIRD) des unteren Respirationstrakts, die größtenteils für die beobachtete Häufung respiratorischer Beschwerden bei Adipositas verantwortlich ist.

\section{- Psychische und soziale Folgen}

Adipositas wird von der Gesellschaft gerne als das Ergebnis von Bequemlichkeit und mangelnder Willenskraft angesehen. Bereits Kindergartenkinder haben ein negatives Bild adipöser Personen verinnerlicht. Zeigt man Kindern Bilder von normalgewichtigen und übergewichtigen sowie behinderten Kindern, so beurteilen sie die übergewichtigen als am unbeliebtesten.

Adipöse Kinder haben ein geringes Selbstwertgefühl. Sie leiden unter Hänseleien ihrer Altersgenossen und der sozialen Isolation. Rund ein Fünftel aller adipösen Kinder sind ängstlich, depressiv und haben soziale Probleme.

Im Jugendalter werden soziale und wirtschaftliche Benachteiligungen offenkundig: Adipöse Jugendliche bekommen schlechtere Ausbildungsplätze und verdienen weniger. Adipöse Mädchen finden seltener einen Partner.

Merke: Der Leidensdruck adipöser Kinder ergibt sich meist aus den psychosozialen Konsequenzen und nicht aus den somatischen Folgeerkrankungen, die meist asymptomatisch sind.

\section{Prävention der Adipositas im Kindes- und Jugendalter}

Da das Körpergewicht vom Bewegungs- und Ernährungsverhalten beeinflusst wird, erscheint es zunächst plausibel, diese Verhaltensweisen durch Wissensvermittlung und/oder Verhaltenstherapie verändern zu wollen. Das (Gesundheits-)Verhalten wird jedoch nur zum Teil von unserem Wissen beeinflusst. Umgebungsfaktoren wie Preis, Werbung und Verfügbarkeit von Lebensmitteln spielen eine genauso wichtige Rolle für das kindliche Ernährungsverhalten, wie die Bewegungsmöglichkeiten für Kinder (z. B. Sicherheit des Schulwegs, zeitliche Freiräume für Bewegung, allgegenwärtige Verfügbarkeit von Fernsehen und Medien) [16]. Alle diese Faktoren entziehen sich jedoch ganz oder weitgehend einem verhaltenstherapeutischen Zugang oder einer Gesundheitserziehung. Daher kann ein verhaltenstherapeutisches Konzept immer nur bedingt Einfluss auf unser Körpergewicht nehmen.

Merke: Randomisierte Präventionsstudien in Kindergärten, Schulen und Gemeinden mit hoher Teilnehmerzahl und guter methodischer Qualität zeigen meist keinen langfristigen Effekt bezogen auf den BMI. Erfolgversprechend sind nach einer aktuellen Cochrane-Review nur Projekte mit Einbindung der Eltern [17].

Nichtkognitive Ansätze sind daher in der Prävention (Verhältnisprävention statt Verhaltensprävention) unbedingt erforderlich. Beispiele hierfür sind Werbeverbot für Lebensmittel in Kindersendungen, Verbot von Getränkeautomaten mit gesüßten Getränken in Schulen oder Sonderabgaben für bestimmte Lebensmittel (z.B. „Kinderlebensmittel“ oder Junk Food). Ein Beispiel für eine effektive verhältnispräventive Maßnahme ist die Trink-Dich-Fit-Studie, bei der in einer randomisierten Clusteranalyse gezeigt werden konnte, dass durch Verbannung von gesüßten Getränken aus Schulen die Prävalenz und Inzidenz von Übergewicht gesenkt werden kann [18]. Weitere sinnvolle Präventionsansätze sind:

Präventionsansätze bei Adipositas

- Verhinderung von Rauchen in der Schwangerschaft

- Stillen

- Verhinderung einer raschen Gewichtszunahme bei Small-for-Gestational-Kindern in den ersten Lebensmonaten

- ausreichender Schlaf

- Reduktion von gesüßten Getränken

- Reduktion von Fast Food

- Reglementierung des Medienkonsums

- Reduktion der Proteinzufuhr in Flaschennahrung 


\section{Lebensstilintervention bei Adipositas im Kindes- und Jugendalter}

Schon eine geringe Gewichtsreduktion reicht aus, um eine Verbesserung der medizinischen Komorbidität der Adipositas zu erreichen. Hierfür genügt es bei wachsenden Kindern, einen Gewichtsstillstand von $1 \mathrm{Jahr}$ zu erzielen, was einer Reduktion des BMI von $1-2 \mathrm{~kg} / \mathrm{m}^{2}$ entspricht [19]. Auch die Intima-Media-Dicke der A. carotis communis nimmt bei diesem Maß der Gewichtsreduktion ab [20].

Die Behandlung adipöser Kindern und Jugendlichen besteht vor allem darin, das Ess- und Bewegungsverhalten langfristig positiv zu beeinflussen. Einfache Ernährungs- und Bewegungstipps, basierend auf den Empfehlungen der AGA, können der folgenden Infobox entnommen werden [3]. Viele weitere Tipps finden sich in entsprechenden Elternratgebern (z. B. [21]).

Bei jeder Anleitung zur Lebensstilveränderung ist jedoch darauf zu achten, die adipösen Kinder und ihre Familien einfühlend zu behandeln und Ratschläge und direktive Handlungsanweisungen, die schnell als Vorwurf missverstanden werden können, zu vermeiden. Zwar sind Ratschläge gut gemeint, allerdings stellen sie schnell eine Kommunikationssituation auf „ungleicher Augenhöhe“ her. Das Arzt-Patienten-Gespräch sollte nicht arztzentriert sein (Abb.5), sondern zwischen gleichberechtigten Partnern stattfinden (klientenzentriertes Gespräch, Abb.6).

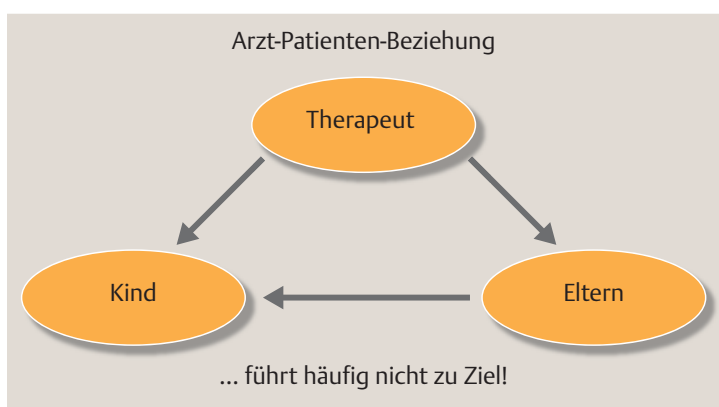

Abb. 5 Arztzentriertes Gespräch: Arzt (und Eltern) sagen, was das Kind zu machen hat.

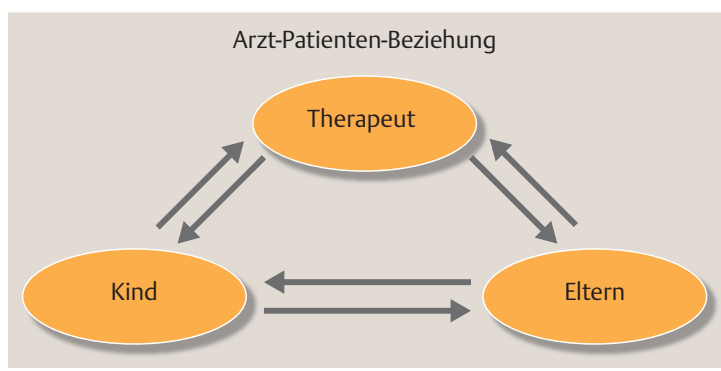

Abb. 6 Klientenzentriertes Gespräch: Eltern und Kind folgen nicht nur den Anweisungen des Arztes, sondern treten gleichberechtigt untereinander in Kommunikation.

Merke: Primär sollte die Familie und nicht der Therapeut Ziele und Therapievorschläge einbringen.

Häufig ist den Familien bewusst, dass das Ernährungsund Bewegungsverhalten verändert werden sollte. Trotzdem gelingt dies den Familien nicht. Ratschläge können so zu Frustration und Überforderung führen. Veränderungen bedeuten jedoch immer ein gewisses Maß an Instabilität und Verunsicherung. Um dem gegenübertreten und dies aushalten zu können, braucht es möglichst viel Selbstvertrauen und Motivation. Der Patient bzw. die Familie muss an sich und

\section{Tipps für Eltern übergewichtiger und adipöser Kinder}

\section{Fernsehkonsum:}

- Stellen Sie keinen Fernseher in das Kinderzimmer.

- Beschränken Sie die Fernseh- und Computerzeiten auf insgesamt maximal 1 Stunde am Tag.

- Gehen Sie mit Ihrem Kind die Fernsehzeitung durch und suchen Sie gemeinsam eine passende Sendung aus.

- Vereinbaren Sie mit Ihrem Kind, dass es den Fernseher nach einer gesehenen Sendung selbständig ausschaltet.

- Bieten Sie in Ihrer Familie Alternativen zum Fernsehprogramm an, wie Gesellschaftsspiele, Gespräche und Vorlesen.
Empfehlungen zur Ernährung:

- Wählen sie ausgewogene und abwechslungsreiche Lebensmittel.

- Naschen und snacken Sie weniger häufig - dafür aber auf höchstem Niveau und mit Genuss. Meiden Sie Vorräte!

- Legen Sie Wert auf regelmäßige, gemeinsame Mahlzeiten in der Familie.

- Essen Sie langsam und mit Genuss.

- Bereiten Sie die Mahlzeiten selbst zu, verwenden Sie so wenige Fertigprodukte wie möglich.

- Essen Sie nur an einem Platz und vermeiden Sie das Essen beim Fernsehen.

- Trinken Sie viel (Mineral-)Wasser und ungesüßte Tees statt energiereiche/ gesüßte Getränke wie Limonaden. Ver- dünnen Sie Fruchtsäfte 1 Teil Saft, 2 Teile Wasser.

- Setzen Sie keine Nahrungsmittel oder Süßigkeiten als Belohnung ein.

- Bieten Sie gesunde Zwischenmahlzeiten (z. B. Obst und Gemüse) als Alternative zu Süßigkeiten an.

- Besuchen Sie Fast-Food-Restaurants nicht häufiger als einmal pro Woche!

- Meiden Sie Großpackungen und XXLAngebote.

- Versuchen Sie angelerntes Fehlverhalten (zu süß essen, zwischendurch essen o. ä.) langsam umzugewöhnen.

- Schauen Sie vor allem bei Kinderprodukten kritisch auf die Inhaltsstoffe. 
seinen/ihren Ressourcen und Stärken glauben, daran, dass es einem selbst möglich ist, das Ziel zu erreichen, etwas zu verändern.

\section{Günstige Gesprächstechniken zur}

Förderung von Veränderungsmotivation

- Empathie zeigen

- Schwerpunkt auf Positivem

- Reframing

- zirkuläre Fragen

- Skalierungsfragen

- Ambivalenzen und Diskrepanzen zulassen

Erläuterungen und Beispiele hierzu finden sich bei den Gesprächsbeispielen im folgenden Kapitel.

Um die Veränderung der Lebensstilgewohnheiten zu unterstützen, ist ferner eine motivierende Gesprächsführung sinnvoll (Abb.7). Bei der motivierenden Gesprächsführung ist es hilfreich, das (Problem-)Verhalten als einen Lösungsversuch zu sehen und - für den Moment - zu akzeptieren, als das, was den Patienten und Familien bisher in den jeweiligen Lebenskontexten möglich war. Gleichzeitig ist davon auszugehen, dass Patienten und Familien motiviert sind, weil sie selbst zumindest in Teilen ein Problem wahrnehmen und eine diesbezügliche Veränderung wünschen - bei der Unterstützung benötigt wird.

Merke: Bei der motivierenden Gesprächsführung trägt nicht der Therapeut, sondern das Patientensystem die Hauptverantwortung für die Veränderung.

Die Familie muss die Veränderungen im eigenen Alltag, tagtäglich, schaffen. Diese Aufgabe wird die Familie nur in ihrem eigenen Tempo und ggf. in ihrer eigenen, möglich und sinnig erscheinenden Abfolge erfüllen können. Letztlich kann nur der Patient bzw. das Familiensystem für sich wissen und entscheiden, was wann der erste bzw. nächste Schritt ist. Dabei ist der Therapeut in der Rolle eines Begleiters, dem es im Idealfall gelingt, (neue) Handlungsspielräume mit zu eröffnen.

Merke: Zentrale Ziele im Rahmen der Gesprächsführung bei Adipositas sind die Förderung von Veränderungsmotivation und die Unterstützung bei der Festlegung konkreter (Teil-)Ziele.

Ziele sollten konkret, präzise und verhaltensbezogen, sein. So wird die Umsetzung im Alltag erleichtert, wenn bereits klar ist, was genau jemand wann, wie, wo und mit wem machen möchte.

- Ziele sollten für das Individuum bedeutsam sein, d. h., es geht um etwas, das wichtig ist, das sich für die jeweilige Person oder Familie zu erreichen lohnt.

- Ziele sollten realistisch sein. Die Chance, dass die gesetzten Ziele erfolgreich erreicht werden können, sollte so groß wie möglich sein. In der Regel lässt sich nicht alles in kürzester Zeit sofort und beständig erreichen.

- Sich zu viel vorzunehmen, führt zwangsläufig zu Frustration. Ziele sollten daher kleinschrittig sein.

\section{Belohnungen}

Insbesondere Belohnungen helfen bei der Umsetzung von (Teil-)Zielen. Es gibt für Eltern sehr viele Möglichkeiten, ein Kind zu belohnen, z. B. zu loben oder das Kind zu umarmen. Gerade das Loben kann, wenn es ernst gemeint und konkret formuliert wird, nicht oft genug erfolgen. Zunächst sehr unangenehme, schwierige Verhaltensänderungen lassen sich auch über Belohnungssysteme trainieren. Wichtig ist, dass die

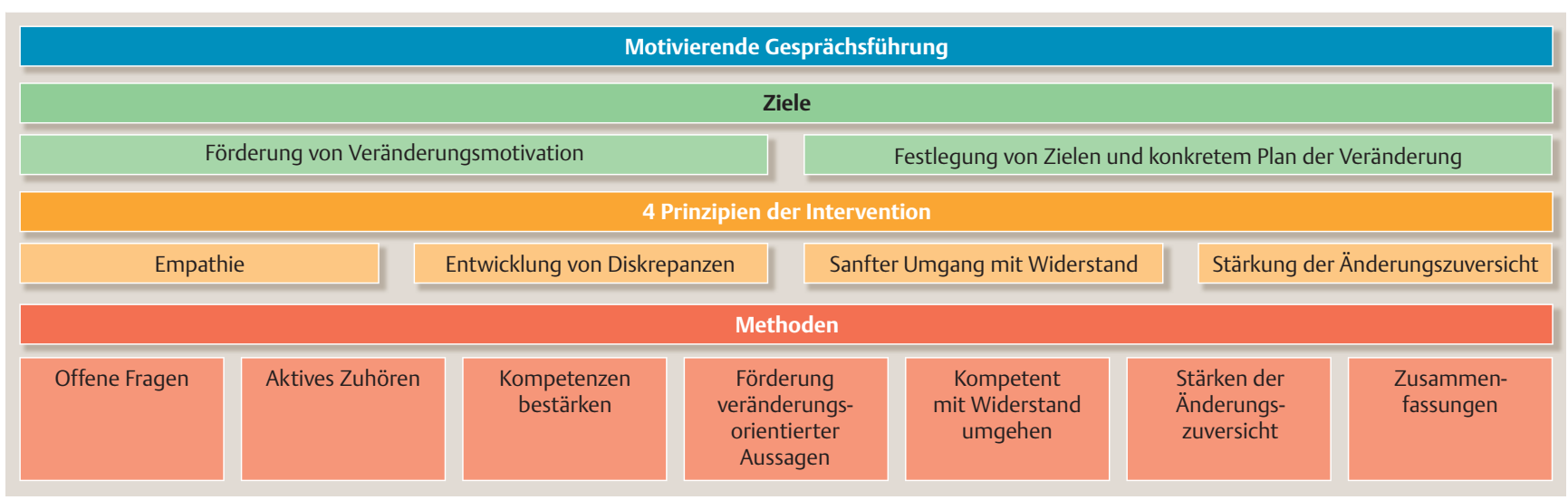

Abb.7 Methoden, Prinzipien und Ziele der motivierenden Gesprächsführung. 
Eltern die Belohnung gemeinsam mit dem Kind aussuchen, so dass diese tatsächlich einen guten Anreiz darstellt. Die Belohnung sollte nicht auf anderen Wegen für das Kind leicht erreichbar sein. Selbstverständlich sollten adipöse Kinder nicht mit Lebensmitteln belohnt werden.

\section{Umgang mit „Widerstand“}

Das Thema Adipositas an sich ist in der Regel belastend und „unangenehm“ für die Betroffenen. Eine Thematisierung und Offenlegung von Diskrepanzen kann darüber hinaus zusätzlich von Kindern und Eltern als konfrontativ erlebt werden. Wenn Menschen das Gefühl bekommen, schlecht dazustehen oder angegriffen zu werden, verwenden sie gerne Umgehungsstrategien, die in manchen Kontexten als „Widerstand“ bezeichnet werden. Zum Beispiel beginnen Kinder/ Jugendliche oder Eltern zu argumentieren, es werden Ausreden vorgebracht, „Ja-aber“-Antworten gegeben, Schuldzuweisungen vorgenommen.

\section{Mögliche Umgehungsstrategien}

\section{bei Widerstand}

- Gehörtes reflektieren und z. B. Ambivalenzen empathisch widerspiegeln.

- Den Gesprächsfokus verschieben (z. B. auf Dinge, die gut klappen, die kohärent sind).

- Herausstellen der persönlichen Wahlfreiheit: Es geht nicht um Wünsche/Ziele des Therapeuten, sondern allein um die Wünsche/Ziele der Kinder und Familien.

- Reframing (siehe Gesprächsbeispiele)

- Konform gehen, d. h. die Kinder/Eltern in ihrem Widerstand bestärken (und so ggf. eine Art Widerstand gegen den Widerstand provozieren).

\section{Gesprächsbeispiele}

Im Folgenden wird zunächst jeweils ein eher negatives Beispiel einer Gesprächsführung dargestellt, mit einer Erklärung, was eine solche Formulierung auslösen kann, gefolgt von einem Beispiel, wie die Formulierung besser ausfallen könnte. Hierbei werden bewusst sehr negative Formulierungen vorgestellt, um für die Themen zu sensibilisieren.

\section{Therapeutische Haltung}

\section{- Neutralität}

Die therapeutische Haltung sollte neutral sein, das heißt, Schuldzuweisungen jeder Arzt sind in jedweder Formulierung zu vermeiden.

Negativbeispiel. „Durch Luft kann man nicht zunehmen, Übergewicht entsteht dann, wenn man sich für seine Ernährungsgewohnheiten zu wenig bewegt.“

Diese Aussage ist wissenschaftlich richtig, beinhaltet aber den (latenten) Vorwurf zu viel zu essen und sich zu wenig zu bewegen. Adipöse Kinder und deren Eltern sind sehr sensibel für dieses Thema, da sie sich alläglich entsprechenden Vorwürfen ausgesetzt sehen. Aus Wahrnehmung der Kinder und Eltern unternehmen diese bereits alles für sie Mögliche, um weniger zu essen und sich mehr zu bewegen. Sie suchen Rat, da sie nicht wissen, was sie noch anders machen können.

Positivbeispiel. Eine andere Formulierung wäre: „Durch den wesentlichen Anteil der Vererbung beim Gewicht ihres Kindes muss der Lebensstil viel perfekter als bei anderen Kindern sein."

Mit einer solchen Formulierung wird eine Therapie eher angenommen, da nicht mit dem Vorwurf gestartet wird, zu viel zu essen und sich zu wenig zu bewegen, sondern mit Verständnis, dass es die Familie sehr schwer hat. Diese Formulierung deckt sich häufig mit den Erfahrungen der Eltern, dass das schlanke Nachbarskind viel mehr isst als der eigene betroffene Nachwuchs.

\section{- Annahme der Motivation}

Es sollte immer eine Motivation der adipösen Kinder und ihrer Eltern angenommen werden, da diese ansonsten gar nicht zu Ihnen gekommen wären.

Negativbeispiel. „Haben Sie schon etwas gegen das Übergewicht Ihres Sohnes unternommen?“

Diese Formulierung beinhaltet wieder den (latenten) Vorwurf, bisher nichts unternommen zu haben.

Positivbeispiel. Eine Alternative wäre folgende Formulierung: „Wie haben Sie gemerkt, dass Ihr Sohn etwas gegen das Übergewicht unternimmt? Wer in der Familie hat dies noch bemerkt?“ 
Dies zeigt, dass der Therapeut der Familie bereits Motivation sowie Kompetenz und Problemlösefertigkeiten unterstellt.

\section{Therapeutische Allianz, Begleiter statt Ratschlag-Geber}

Die Rolle des Therapeuten in einem gleichberechtigten Gesprächsmodell (Abb.6) liegt in der Begleitung und nicht in der Aufgabe, Ratschläge zu geben (Abb.5).

Negativbeispiel. „Ich schlage vor, dass Sie den Fernsehkonsum ihres Sohnes reduzieren.“

Dieser Ratschlag ist gut gemeint, aber trotzdem ein „Schlag“ für die Familie. Diese Formulierung beinhaltet den (latenten) Vorwurf, dies bisher noch nicht gemacht zu haben. Ratschläge sind immer auch „Schläge“. Jede direkte Handlungsanweisung löst Widerstand aus, und man findet schnell viele Gründe, warum dies nicht umsetzbar ist.

Positivbeispiel. Alternative Formulierungen (mit demselben Inhalt) wären z. B.: „Einige Studien haben gezeigt, dass Kinder bei Reduktion des Fernsehkonsums Gewicht verlieren können. Empfehlungen sprechen von maximal 2 Stunden Fernsehen pro Tag. Was denken Sie darüber?“ oder noch besser: „Einige Familie erzählen, dass wenn ein Frühstück eingenommen wird, die Kinder weniger Hunger haben. Wie denken Sie darüber?"

Durch diese Formulierungen wird der Therapeut zum Begleiter der Familie. Der Therapeut überlegt gemeinsam mit der Familie, ob dieser Vorschlag zur Familie passt. Die Eltern und Kinder fühlen sich hierdurch weniger unter Druck gesetzt und gehen nicht direkt in einen Ablehnungsmodus über.

\section{Den Fokus auf Stärken legen}

In der Adipositastherapie neigen Therapeuten häufig dazu, Probleme (Schwächen) hervorzuheben und hier nach Änderungsmöglichkeiten zu suchen. Somit dreht sich schnell das Gespräch nur um Negatives, und dies ist für Keinen angenehm. Besser ist es, die Stärken herauszustellen und diese zu benutzen, um Herausforderungen zu überwinden.
Negativbeispiel. Therapeut: „Frühstückst Du?“- Kind: „Ja, gelegentlich.“-Therapeut: „Warum frühstückst Du nicht jeden Tag?“

Diese Formulierung fokussiert auf den Misserfolg, und die Behandlung wird damit schnell aus Sicht des Kindes mit Negativem besetzt. Das Selbstbewusstsein des Kindes wird zudem herabgesetzt.

Positivbeispiel. Eine alternative Formulierung könnte lauten: „Wie viele Tage die Woche frühstückst Du? Was ist besonders an den Tagen, an denen du frühstückst?“

Nun wird der Fokus auf die Stärken gelegt und überlegt, was unter Umständen an den Tagen besonders ist, an denen es gelingt zu frühstücken.

\section{Empathie ausdrücken}

In Gesprächen Empathie auszudrücken, ist manchmal eine Herausforderung, insbesondere, wenn man sich durch Jugendliche oder auch Eltern angegriffen fühlt.

\section{Reaktion auf „Angriffe“}

Häufig steht hinter einem „gefühlten“ Angriff der Patienten der Ruf nach Aufmerksamkeit. Ein Vater könnte etwa, nachdem der Therapeut etwas vorgestellt hat, äußern: „Ich habe die neuesten Forschungsergebnisse dazu gelesen. Ich bin auf dem aktuellsten Stand.“

Negativbeispiel. Therapeut: „Ich bin der Fachmann. Sie können sich darauf verlassen, dass alle unsere Vorschläge Stand der Wissenschaft sind.“

Hiermit geht der Therapeut auf Konfrontationskurs und stellt sein Wissen über das Wissen des Vaters. Dies ist häufig der Beginn eines konfliktreichen Gesprächs.

Positivbeispiel. Eine alternative Reaktion könnte so aussehen: „Ich merke, Sie haben sich intensiv mit dem Thema ausgesetzt, das zeigt, wie motiviert Sie sind.“

Der Therapeut zollt dem Vater Wertschätzung. Dadurch fühlt sich der Vater eher verstanden und Widerstände können sich auflösen.

\section{Reaktion auf "Hilferufe“}

Aussagen wie: „Ich bin alleinerziehend. Es ist alles so schwierig, niemand unterstützt mich." beinhalten unter Umständen neben der Klage auch einen Hilferuf nach Unterstützung sowie den Ruf nach Anerkennung. Wenn es dem Therapeuten geling, darauf zu reagieren 
und daran anzuknüpfen, wird die Beziehungsebene zwischen Therapeut und Elternteil gestärkt.

Positivbeispiel. Ein Beispiel einer empathischen Äußerung des Therapeuten könnte sein: „Ich merke, dass auf Ihnen eine große Verantwortung lastet und Sie ganz Unglaubliches leisten. Da wünschen Sie sich manchmal mehr Unterstützung ...“

\section{Wunsch nach Autonomie}

Aussagen von Jugendlichen wie: „Ich will meine Ruhe. Die sollen auch nicht einfach immer in mein Zimmer kommen!" können unter anderem anzeigen, dass der Jugendliche sich mehr Autonomie wünscht.

Positivbeispiel. Eine empathische Formulierung des Therapeuten könnte sein: „Wenn ich Dich recht verstehe, möchtest Du weniger durch die Erwachsenen kommandiert werden und stattdessen selbst für Dich Verantwortung übernehmen?“

\section{Reframing}

Wenn es dem Therapeuten gelingt, nicht nur empathisch zu sein, sondern dem Gesagten auch noch eine neue Deutungsebene hinzuzufügen, eröffnet sich die Möglichkeit, dass die Familien neue Verhaltensstrategien entwickeln. Die Mutter sagt zum Beispiel: „Ich weiß nicht, wie ich meiner Tochter helfen soll. Ich muss den ganzen Tag schimpfen, ich bin wie ein Kassettenrecorder von morgens bis abends."

Negativbeispiel. Therapeut: „Ermahnungen führen zu nichts, besser ist es, das Positive zu sehen.“

Hier wertet der Therapeut und gibt direktive Handlungsanweisungen, die leicht zu Widerstand führen können.

Positivbeispiel. Besser wäre z.B. die Formulierung: „Sie fragen sich, ob es hilfreich ist, Ihre Tochter immer wieder zu ermahnen?“

Hier wiederholt der Therapeut das Gesagte mit anderen Worten, um zum einen Verständnis zu zeigen und zum anderen Ansatzmöglichkeiten für eine neuen Deutungskontext zu erarbeiten.

\section{Zielfestlegung}

Um konkrete Handlungsperspektiven zu erarbeiten, ist es zunächst wichtig, herauszufinden, ob die Familie eigene Veränderungsmöglichkeiten sieht. Nach der offenen Frage durch den Therapeuten, wie es in der letzten Zeit gelaufen ist, kommen bei motivierten Familien häufig schon Vorschläge vom Kind/Jugendlichen oder von den Eltern, was anders laufen könnte. Der Jugendliche könnte z. B. als Vorsatz nennen, dass er nun mit dem Fahrrad in die Schule fahren will.

Negativbeispiel. Eine eher ungünstige Formulierung des Therapeuten wäre: „Das ist eine super Idee. Beim nächsten Mal erzählst Du mir, wie es gelaufen ist.“

Schwierigkeiten in der Durchführung werden hier nicht bedacht. Wenn das Ziel nicht erreicht wird, befürchtet das Kind unter Umständen, dass der Therapeut enttäuscht sein könnte und vermeidet es, wiederzukommen, um sein „Versagen“ nicht publik machen zu müssen. Eine dauerhafte Verhaltensmodifikation ist dann sehr schwierig. Die Ziele sollten möglichst konkret sein. Ohne konkrete, überprüfbare Ziele, die auch realisierbar sind, ist die Wahrscheinlichkeit groß, dass das erwünschte Verhalten nicht dauerhaft eintritt. Aus diesen Gründen erscheint es besser, Ziele bezüglich ihrer Realisierbarkeit zu hinterfragen und möglichst kleinschrittig fest zu setzen.

Positivbeispiel. Auf den Vorschlag des Jugendlichen, mehr mit dem Fahrrad zur Schule zu fahren, könnte der Therapeut z. B. einwerfen: „Das ist eine super Idee, aber eventuell wird es auf Dauer sehr schwierig. Was machst Du, wenn es regnet? Ich schätze es als sehr schwierig ein, dann mit dem Fahrrad zur Schule zu fahren.“

Hierbei wird der Vorschlag aufgriffen und negiert bzw. relativiert, und dabei werden denkbare Hinderungsgründe genannt. Dies steigert häufig auch den Ehrgeiz der „Betroffenen“.

Wichtig ist es, immer nur mit einem Vorschlag zu arbeiten und konkrete Handlungsvorschläge zu erarbeiten. Diese können z.B. auch vertraglich in der Akte vereinbart und vom Jugendlichen unterschrieben werden, was ebenfalls den Ehrgeiz steigert. Wichtig ist natürlich, bei der nächsten Vorstellung unbedingt nach der Vereinbarung zu fragen. 


\section{Umgang mit Widerstand}

Ist die Familie resigniert, kann neben der motivierenden Gesprächsführung eine andere Herangehensweise helfen.

Negativbeispiel. Auf die Aussage der Mutter: „Wir haben wirklich alles probiert.“ in der folgenden Form zu antworten, erscheint eher ungünstig: „Haben Sie denn schon darüber nachgedacht, den Jungen in einem Sportverein anzumelden?“

Abgesehen davon, dass adipöse Kinder in Sportvereinen häufig nur Misserfolge erleben, wird eine direktive Handlungsanweisung leicht zu Widerstand und Widerspruch führen.

Positivbeispiel. Alternativ könnte man erwidern: „Ja da kann man wirklich nichts machen. Sie haben wirklich alles probiert.“

In der Regel regt sich dann Widerstand durch Eltern oder das Kind, so dass wieder Handlungsspielraum entsteht und Vorschläge von der Familie selbst erarbeitet werden. Zur Not kann man auch nachfragen: „Wer hat Einfluss auf das Gewicht von Hans?“ oder die „Sinnfrage“ stellen: „Was hast Du davon abzunehmen?“ Wenn kein Vorteil gesehen wird, abzunehmen, ist zurzeit offenbar nicht der richtige Zeitpunkt für eine Lebensstilmodifikation.

\section{Suche nach der Motivation}

Wenn nach Motivation zur Gewichtsabnahme gesucht wird, sollte man für die Kinder und Jugendliche spürbare Vorteile suchen.

Negativbeispiel. Therapeut: „Wenn du nicht bald Gewicht abnimmst, wirst du gesundheitliche Schäden davontragen.“

Fast alle Folgeerkrankungen der Adipositas sind für die Jugendlichen nicht spürbar und erzeugen eher Angst (z.B. wurde beim Großvater aufgrund eines Diabetes eine Beinamputation durchgeführt und das Kind hat nun mehr Sorge, dass es auch die Beine verlieren wird, als dass es durch die Adipositas vom Diabetes bedroht ist).
Positivbeispiel. Besser ist es, auf die Tragbarkeit von Kleidung, weniger Hänseleien oder verbesserte sportliche Belastbarkeit als Vorteil einer Gewichtsabnahme hinzuweisen.

Tipps für die Gesprächsführung bei

Kindern/Jugendlichen mit Adipositas

Generelle Haltung: Jede Familie, die sich in der Sprechstunde vorstellt, ist motiviert.

- Fokus auf Stärken legen, nicht auf Schwächen.

- Keine Ratschläge.

- Wenn der Therapeut mehr arbeitet als die Familie, läuft etwas verkehrt.

- Immer nur ein Ziel, kleinschrittig arbeiten.

- Nicht gegen Widerstand argumentieren.

- „Widerstand“ als Hinweis auf Probleme in der Gesprächsführung ansehen.

\section{Weiterführende Behandlung}

Sollte eine Anleitung zur Lebensstilveränderung in der oben beschriebenen Form nicht zu einem Erfolg führen, ist entsprechend internationalen und nationalen Leitlinien eine langfristige, ambulante, verhaltenstherapeutisch orientierte Schulung bestehend aus einer Ernährungs-, Verhaltens- und Bewegungstherapie unter Einbeziehung der Eltern Therapie der Wahl $[1,17]$.

Merke: Die publizierten Studien zeigen übereinstimmend, dass nur bei motivierten Teilnehmern und intensiver Einbindung der Eltern mit einem langfristigen, multidisziplinären Therapieansatz ein Erfolg erzielt werden kann [17, 22].

Die entsprechenden Leitlinien der Arbeitsgemeinschaft Adipositas im Kindes- und Jugendalter (AGA) und der Deutschen Adipositasgesellschaft (DAG) sind auf den folgenden Seiten zusammengefasst (s. Infobox). Ein Adressverzeichnis von zertifizierten Therapieeinrichtungen für adipöse Kinder und Jugendliche in Deutschland sowie Leitlinien zur Behandlung finden sich unter www.a-g-a.de. 


\section{Grenzen von Schulungsprogrammen}

Nur eine Untergruppe der adipösen Kindern und Jugendlichen spricht auf ein strukturiertes Schulungsprogramm an [23]. Ursachen für Schwierigkeiten bei der Gewichtsabnahme sollten nicht nur bei dem Kind oder bei der fehlenden Motivation der Familie gesucht werden, sondern sind auch durch den genetischen Hintergrund und die hormonellen Gegenregulationsmechanismen nach der Gewichtsabnahme bedingt [22] (Abb. 8). Daher sind Vorwürfe bei Misserfolg nicht angebracht.

Geistig retardierte Kinder, körperlich behinderte Kinder, Kinder mit fehlender Gruppenfähigkeit, extrem adipöse Jugendliche und unmotivierte Kinder und Familien sind einer Lebensstilintervention meist nicht zugänglich [22]. Bisher sind keine langfristig wirksamen Behandlungskonzepte basierend auf einer Lebensstilintervention für diese Patientengruppen entwickelt worden. Insbesondere bei Folgeerkrankungen der Adipositas sollten weitere Behandlungsoptionen erwogen werden.

\section{Medikamentöse Therapie}

Die medikamentöse Behandlung der kindlichen Adipositas zeigt nur eine relativ moderate Effektivität, ähnlich der von Schulungsprogrammen. Einzelne Präparate (z. B. Rimonabant oder Sibutramin) sind aufgrund potenziell lebensgefährlicher Nebenwirkungen vom europäischen Markt genommen worden. Somit bleibt für das Kinderalter nur ein zugelassenes Medikament, der Lipasehemmer Orlistat. Das Nebenwirkungsprofil (Fettstühle beim Verzehr größerer Mengen Fett) kann als „Biofeedback“ zur Unterstützung der Ernährungsumstellung dienen.

Insgesamt ist von Medikamenten jedoch kein dauerhafter Erfolg zu erwarten, da:

- Nebenwirkungen die Compliance senken,

- Medikamente lebenslang genommen werden müssten,

- Medikamente nicht zu einer Verhaltensänderung führen und

- nur geringe Gewichtsveränderungen mit Medikamenten zu erzielen sind.

Merke: Insgesamt ist von Medikamenten kein dauerhafter Erfolg zu erwarten.

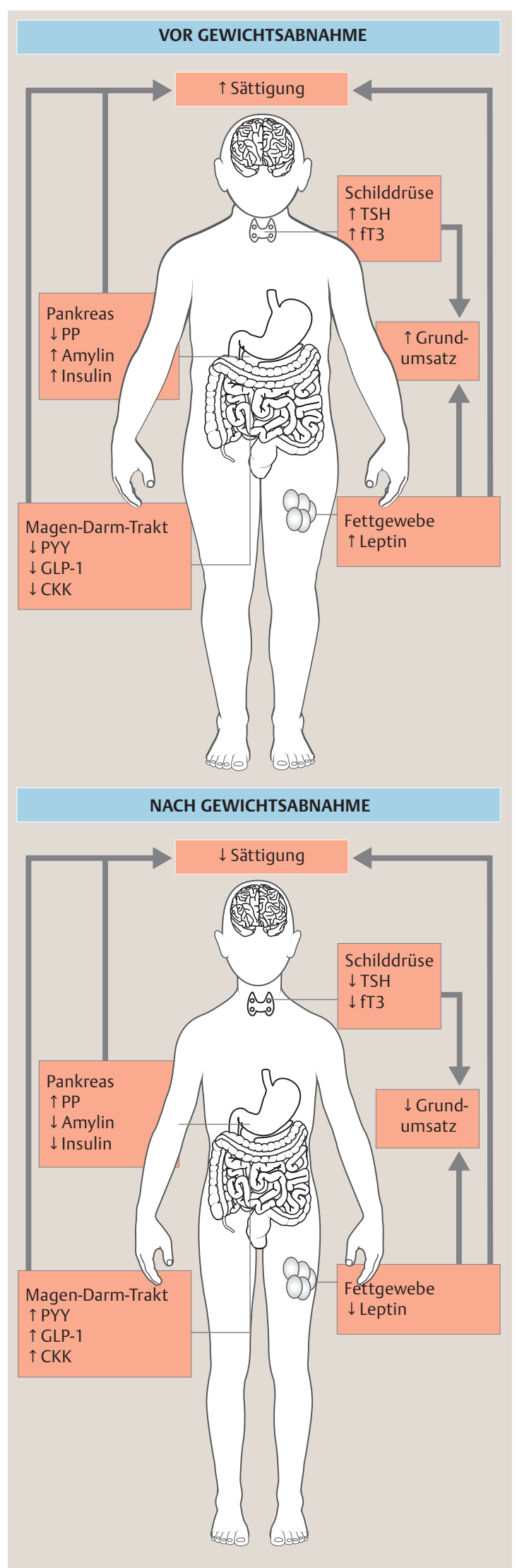

Abb. 8 Hormonelle Gegenregulation bei Gewichtsabnahme adipöser Kinder; Kompensationsmechanismen bei adipösen Kindern vor und nach Gewichtsabnahme. Sättigende Hormone sind Leptin, Amylin, PP, PYY, CKK, GLP-1 und Insulin (modifiziert nach [22]). PP: pankreatisches Polypeptid PYY: Polypeptid YY GLP-1: Glucagonlike Peptid 1 CKK: Cholecystokinin TSH: Thyreoidea stimulierendes Hormon fT3: freies Triiodthyronin 
Kernaussagen der Leitlinien der Arbeitsgemeinschaft Adipositas im Kindes- und Jugendalter (AGA) und der Deutschen Adipositasgesellschaft (DAG) [3]

- Grundlage einer Adipositastherapie in jeder Altersstufe sollte ein multimodales Programm sein, das die Komponenten Ernährungs-, Bewegungs- und Verhaltenstherapie umfasst, da isolierte Ernährungs-, Bewegungs- und Verhaltenstherapien nicht zu einem langfristigen Erfolg führen.

- Im Kindesalter müssen die Eltern bzw. die Familie oder die Betreuer des Patienten eine neben dem Patienten gleich bewertete Zielgruppe für die Therapie sein (familienbasierte Therapie).

- Die Familie sollte motivierend und unterstützend im Rahmen der Adipositasbehandlung mitwirken.

- Starre Diätpläne oder Kostformen mit extremen Nährstoffrelationen (z. B. häufige Gewichtsreduktionsdiäten, totales Fasten, „Heilfasten“, Schrothkur, MayrKur, Ananasdiät etc.) sollten wegen potenzieller medizinischer Risiken und fehlendem Langzeiterfolg nicht angewandt werden.
- Die Steigerung der körperlichen Aktivität sollte im Gruppensetting erfolgen, da hier neben der körperlichen Aktivität gleichzeitig die gegenseitige Motivation gestärkt wird.

- Primäre Ziele der Bewegungstherapie sind die Verringerung der körperlichen Inaktivität (z. B. Medienkonsum, TV/ Computer), die Steigerung der Alltagsaktivität und die Anleitung zum körperlichen Training.

- Die Steigerung der körperlichen Bewegung im Alltag soll primäres Ziel einer Bewegungstherapie sein. Sie ist langfristig effektiver bezüglich der Gewichtsreduktion als die Teilnahme an zeitlich limitierten Sportprogrammen.

- Bei Adipositas im Kindes- und Jugendalter kann in Einzelfällen eine medikamentöse Therapie zur Übergewichtsreduktion erwogen werden, insbesondere bei Patienten mit erheblicher Komorbidität und einem extrem erhöhten Gesundheitsrisiko sowie Versagen einer herkömmlichen verhaltensorientierten Therapie über mindestens 9-12 Monate.

- Die Effektivität adipositaschirurgischer Maßnahmen bezüglich Gewichtsreduktion ist bei Jugendlichen durch eine Vielzahl von klinischen Studien belegt. Das Risiko für Komplikationen und Nebenwirkungen muss allerdings berücksichtigt werden. Eine chirurgische Maßnahme sollte nur als letzte therapeutische Möglichkeit nach Scheitern sämtlicher konservativer Therapien bei extrem adipösen Patienten mit erheblicher Komorbidität erwogen werden. Adipositaschirurgische Eingriffe sollen in spezialisierten Einrichtungen, die das ganze Spektrum der operativen adipositasspezifischen Techniken sowie auch Rezidiveingriffe anbieten, vorgenommen werden. Die operierten Patienten sollen in einem über den Eingriff hinaus langfristigen, multidisziplinären Konzept betreut werden.

\section{Bariatrische Chirurgie}

Chirurgische Methoden sind im Langzeitverlauf die effektivsten Verfahren zur Gewichtsreduktion. Diese sehr guten Erfolge sind zum Teil auch durch die Veränderung des Hormonprofils in der Sättigungsregulation bedingt. Jedoch haben bariatrische Verfahren eine Vielzahl von schwerwiegenden Nebenwirkungen.

Malabsorptionstechniken wie der jejunoileale Bypass sind auch bei Jugendlichen trotz guter Wirksamkeit verlassen worden, aufgrund der Häufigkeit und Schwere der Nebenwirkungen (toxische Leberschäden, Enzephalopathie, Megakolon, Ileus, Vitamin-B-Mangel).

Die Kombination von Magenrestriktion und Malabsorptionstechniken wie beim Roux-en-Y-MagenBybass ist ein weiteres, sehr erfolgreiches Verfahren bei extrem adipösen Jugendlichen: 5 Jahre nach Operation wurde das relative Übergewicht durchschnittlich um $51 \%$ gesenkt. Auch dieses Verfahren zeigt schwerwiegende Nebenwirkungen wie Magenstenose, Ernährungsstörung (v.a. Eisen- und Folatmangel), Dumping-
Syndrom und Notwendigkeit einer Re-Operation bei einer Mortalität von $5 \%$.

Heutzutage werden aufgrund der geringen Nebenwirkungsrate bei extrem adipösen Jugendlichen vor allem Magenrestriktionen (Gastroplastik, Schlauchmagen) verwendet.

Ein Magenband, welches laparoskopisch eingesetzt werden kann, wird aufgrund der vielen Komplikationen im Verlauf und der begrenzten Wirkung bei extrem adipösen Jugendlichen nicht mehr empfohlen.

Merke: Eine operative Therapie ist nur als Methode der letzten Wahl bei extrem adipösen Jugendlichen aufgrund der Nebenwirkungen und Langzeitfolgen anzusehen. 


\section{Therapie der Folge- erkrankungen}

Wenn keine Gewichtsreduktion zu erzielen ist, müssen die Folgeerscheinungen der Adipositas bereits bei Kindern und Jugendlichen konsequent behandelt werden.

Bluthochdruck. Eine arterielle Hypertonie sollte durch einen pädiatrischen Nephrologen und einen Kinderkardiologen abgeklärt und behandelt werden (s.a. www.hochdruckliga.de). Eine Monotherapie, die in der Regel mit einem ACE-Hemmer durchgeführt wird und keinesfalls mit Betablockern (führen zur Reduktion des Grundumsatzes und erhöhen das Diabetesrisiko), ist in den meisten Fällen effektiv.

Diabetes mellitus. Bei einem manifesten Typ-2-Diabetes wird nach erfolgloser Gewichtsreduktion über 3 Monate ab einem HbA1c>7,0\% eine medikamentöse Therapie mit Metformin begonnen. Metformin wird einschleichend dosiert und zu den Mahlzeiten eingenommen. Trotzdem treten bei ca. $10 \%$ der Fälle gastrointestinale Nebenwirkungen auf. Andere orale Antidiabetika sind bisher nicht für Kinder und Jugendliche zugelassen.

Bestehen bereits klinische Zeichen eines Insulinmangels oder ist die Differenzialdiagnose bezüglich des Diabetes-Typs unklar, sollte im Zweifel mit einer Insulintherapie begonnen werden. Die Therapie von Kindern und Jugendlichen mit Diabetes gehört in die Hände eines pädiatrisch-diabetologisch erfahrenen Teams, da neben einer kontinuierlichen Betreuung eine strukturierte Diabetesschulung notwendiger Bestandteil der Therapie ist.

Weitere Folgeerkrankungen. Orthopädische Erkrankungen (Epiphyseolysis, Genu valgum usw.) und endokrinologische Erkrankungen (Pubertas praecox, polyzystisches Ovarsyndrom) sollten durch entsprechende Spezialisten (Orthopäden bzw. pädiatrische Endokrinologen) behandelt werden.

Ein Schlaf-Apnoe-Syndrom wird mit einer NCPAPMaske versorgt.

\section{Anmerkung}

Teile dieser Arbeit erschienen ebenfalls in Aktuelle Ernährungsmedizin 2015; 2: 102 -122.

\section{Fazit}

Adipositas ist im Kindes- und Jugendalter nach der dramatischen Zunahme in den letzten 20 Jahren seit circa 5 Jahren nicht mehr weiter zunehmend, wohl aber der Anteil extrem adipöser Jugendlicher. Adipositas ist bereits im Kindesalter mit einer Vielzahl von kardiovaskulären Risikofaktoren assoziiert. Während diese meist asymptomatisch sind, leiden die adipösen Kinder v.a. unter den psychosozialen Folgen. Die Ursache der Adipositas im Kindesalter ist meist multifaktoriell und reicht von genetischen Faktoren über Umweltfaktoren bis hin zur familiären Situation. Bisherige Präventionskonzepte sind noch nicht nachhaltig überzeugend. Die Therapie der Wahl besteht aus einer Lebensstilintervention basierend auf Schulungsprogrammen. Bei der motivierenden Gesprächsführung basierend auf den Stärken und nicht Schwächen der Adipösen trägt nicht der Therapeut sondern das Patientensystem die Hauptverantwortung für die Veränderung. Misserfolge sind nicht nur auf fehlende Motivation sondern auch auf die Gegenregulation des Körpers bei Gewichtsabnahme zurückzuführen. Die Therapie der extremen Adipositas im Jugendalter ist ein ungelöstes Problem, da Schulungsprogramme in der Regel nicht erfolgreich sind. Bariatrische Verfahren können zwar effektiv das Ausmaß des Übergewichts reduzieren, haben jedoch vielfältige Nebenwirkungen und benötigen eine lebenslange Betreuung, welche bereits vor Beginn der Operation gewährleistet sein muss.

\section{Über die Autoren}

\section{Caroline Knop}

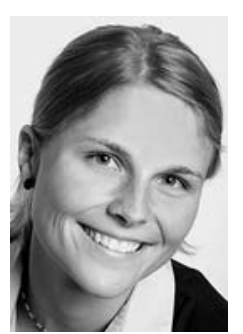

Dr. med. Kinder- und Jugendärztin in der Ausbildung zur Pädiatrischen Endokrinologin und Diabetologin. Frau Knop ist Mitglied der Arbeitsgruppe in der Abteilung für Pädiatrische Endokrinologie, Diabetologie und Ernährungsmedizin an der Vestischen Kinder- und Jugendklinik, Universität Witten/Herdecke, die sich schwerpunktmäßig mit der Diagnostik und Therapie der kindlichen Adipositas beschäftigt.

\section{Thomas Reinehr}

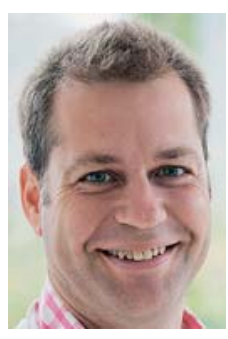

Prof. Dr. med. Kinder- und Jugendarzt, Pädiatrischer Endokrinologe und Diabetologe sowie Pädiatrischer Gastroenterologe. Herr Reinehr leitet die Abteilung für Pädiatrische Endokrinologie, Diabetologie und Ernährungsmedizin an der Vestischen Kinderund Jugendklinik, Universität Witten/ 
Herdecke. Der Forschungsschwerpunkt seines Teams liegt in der kindlichen Adipositas und dem Diabetes mellitus Typ 2. Die Forschungsgruppe wird unter anderem von der Deutschen Forschungsgemeinschaft, dem Bundesforschungsministerium und der Europäischen Union finanziert. Dabei wird der Fokus nicht nur auf Grundlagenforschung gelegt, sondern auf patientenzentrierte Forschung, so dass z.B. ein Schulungsprogramm für adipöse Kinder und Jugendliche „Obeldicks“ entwickelt werden konnte, welches sich in multizentrischen, randomisiert kontrollierten Studien als wirksam erwiesen hat.

\section{Korrespondenzadresse}

Prof. Dr. med. Thomas Reinehr

Abteilung für Pädiatrische Endokrinologie,

Diabetes und Ernährungsmedizin

Vestische Kinder- und Jugendklinik Datteln

Universität Witten/Herdecke

Dr. Friedrich Steiner Str. 5

45711 Datteln

E-Mail:T.Reinehr@kinderklinik-datteln.de

Interessenkonflikt: Die Autoren geben an, dass kein Interessenkonflikt besteht.

\section{Literatur}

1 Ebbeling CB, Pawlak DB, Ludwig DS. Childhood obesity: public-health crisis, common sense cure. Lancet 2002; 360: 473-482

2 Biro FM, Wien M. Childhood obesity and adult morbidities. Am J Clin Nutr 2010; 91: 1499-1505

3 Guidelines of the German working group on obese children and adolescents. 2014: http: www.a-g-a.de/Leitlinie.pdf

4 Kurth BM, Schaffrath RA. The prevalence of overweight and obese children and adolescents living in Germany. Results of the German Health Interview and Examination Survey for Children and Adolescents (KiGGS). Bundesgesundheitsblatt Gesundheitsforschung Gesundheitsschutz 2007; 50: 736 743

5 Moss A, Klenk J, Simon K et al. Declining prevalence rates for overweight and obesity in German children starting school. Eur J Pediatr 2012; 171: 289-299

6 Reinehr T, Hinney A, de Sousa G et al. Definable somatic disorders in overweight children and adolescents. J Pediatr 2007; 150: $618-622$
7 Reinehr T, Dobe M, Winkel K et al. Adipositas bei behinderten Kindern und Jugendlichen Eine therapeutisch vergessene Patientengruppe. Dtsch Arztebl Int 2010; 107: 268-275

8 Kromeyer-Hausschild K, Gläßer N, Zellner K. Waist Circumference percentile in Jena children (Germany) 6 to 18 years of age. Aktuel Ernaehr Med 2008; 33: 116-122

9 Reinehr T, de Sousa G, Andler W. Hyperthyrotropinemia in obese children is reversible after weight loss and is not related to lipids. J Clin Endocrinol Metab 2006; 91: 3088-3091

10 Reinehr T, Kulle A, Wolters B et al. Relationships between 24hour urinary free cortisol concentrations and metabolic syndrome in obese children. J Clin Endocrinol Metab 2014; 99 : $2391-2399$

11 Hinney A, Vogel Cl, Hebebrand J. From monogenic to polygenic obesity: recent advances. Eur Child Adolesc Psychiatry 2010; 19: $297-310$

12 Wunsch R, de Sousa G, Reinehr T. Intima-media thickness in obesity: relation to hypertension and dyslipidaemia. Arch Dis Child 2005; 90: 1097

13 Reinehr T, Andler W, Denzer C et al. Cardiovascular risk factors in overweight German children and adolescents: relation to gender, age and degree of overweight. Nutr Metab Cardiovasc Dis 2005; 15: $181-187$

14 Reinehr T, Kiess W, de Sousa G et al. Intima media thickness in childhood obesity: relations to inflammatory marker, glucose metabolism, and blood pressure. Metabolism 2006; 55: 113 118

15 Reinehr T. Type 2 diabetes mellitus in children and adolescents. World J Diabetes 2013; 4: 270-281

16 Müller J, Reinehr T, Hebebrand J. Prävention und Therapie der Adipositas im Kindes- und Jugendalter. Dtsch Arztebl 2006; 103: $334-340$

17 Oude LH, Baur L, Jansen $\mathrm{H}$ et al. Interventions for treating obesity in children. Cochrane Database Syst Rev 2009: CD001872

18 Muckelbauer R, Libuda L, Clausen K et al. Promotion and provision of drinking water in schools for overweight prevention: randomized, controlled cluster trial. Pediatrics 2009; 123: e661 - e667

19 Reinehr T, Kiess W, Kapellen T et al. Insulin sensitivity among obese children and adolescents, according to degree of weight loss. Pediatrics 2004; 114: 1569-1573

20 Wunsch R, de Sousa G, Toschke AM et al. Intima-media thickness in obese children before and after weight loss. Pediatrics 2006; 118: $2334-2340$

21 Reinehr T, Dobe M, Kersting M. Tipps und Tricks von Obeldicks und Optimix: Der Ratgeber für Eltern übergewichtiger Kinder. Göttingen: Hogrefe; 2009

22 Reinehr T. Lifestyle intervention in childhood obesity: changes and challenges. Nat Rev Endocrinol 2013; 9: 607-614

23 Reinehr T, Widhalm K, I'Allemand D et al. Two-year follow-up in 21,784 overweight children and adolescents with lifestyle intervention. Obesity (Silver Spring) 2009; 17: 1196-1199 


\section{CME-Fragen}

\section{CME.thieme.de}

CME-Teilnahme

- Viel Erfolg bei Ihrer CME-Teilnahme unter http://cme.thieme.de

- Diese Fortbildungseinheit ist 12 Monate online für eine CME-Teilnahme verfügbar.

- Sollten Sie Fragen zur Online-Teilnahme haben, unter http://cme.thieme.de/hilfe finden Sie eine ausführliche Anleitung.

\section{1}

Wodurch entsteht der Leidensdruck bei adipösen Kindern?
A psychosoziale Konsequenzen
B Bluthochdruck
C Fettleber
D Diabetes mellitus Typ 2
E Dyslipidämie

\section{2}

Welches Medikament ist zur Gewichtsreduktion bei Kindern ab 12 Jahren zugelassen?
A Metformin
B Orlistat
C Sibutramin
D Locerasid
E Rimonabant

\section{3}

Welche Aussage trifft nicht zu?
A Die Wirkung einer Lebensstilintervention ist weitgehend vom Geschlecht unabhängig.
B Bei einer Lebensstilintervention müssen die Eltern eingebunden werden.
C Eine Lebensstilintervention sollte sich auf die Ernährung und Bewegung fokussieren.
D Eine Lebensstilintervention ist bei vielen adipösen Kindern wirksam.
E Eine Lebensstilintervention sollte im Rahmen von strukturierten Schulungen durchgeführt werden.

Was ist kein somatischer Grund für Übergewicht im Kindes- und Jugendalter?
A Prader-Willi-Syndrom
B Kraniopharyngeom
C MC4-Rezeptordefekt
D Silver-Russel-Syndrom
E Wachstumshormonmangel

5

Welcher Parameter wird nicht zur Definition eines metabolischen Syndroms verwendet?
A 2-Stunden-Wert im OGTT
B Triglyzeridwert (nüchtern)
C Blutdruckwert
D HDL-Cholesterinwert
E LDL-Cholesterinwert

\section{6}

Welche Aussage zu psychosozialen Konsequenzen der kindlichen Adipositas trifft nicht zu? Adipöse Jugendliche...
A bekommen schlechter einen Ausbildungsplatz.
B finden schlechter einen Partner.
C schreiben schlechtere Noten in der Schule.
D haben ein geringeres Selbstwertgefühl.
E sind häufig ängstlich. 


\section{CME-Fragen}

Diagnostisches und therapeutisches Vorgehen bei Adipositas im Kindes- und Jugendalter

\section{7}

Wie kann die Familie dem Kind helfen, Gewicht zu reduzieren?
A Bestimmte Diäten (z. B. Low Carb) zusammen mit dem Kind durchführen.
B Süßigkeiten nur als Belohnung einsetzen, ansonsten auf diese verzichten.
C Bestimmte Nahrungsmittel und Süßigkeiten verstecken.
D Essen als Nebenbeschäftigung, z. B. während einer Fernsehsendung, damit das Kind es nicht als wichtig empfindet.
E Vermeiden von Vorräten und Großpackungen.

\section{8}

Folgende Untersuchung sollte bei allen adipösen Kindern durchgeführt werden:
A Schlaflaboruntersuchung
B Wachstumshormontestung
C Blutdruckmessung
D oraler Glukosetoleranztest
E Bestimmung des Kortisolwerts

Welche Aussage trifft zu?
A Das Sotos-Syndrom ist mit Adipositas und Kleinwuchs assoziiert.
B Das Bardet-Biedl-Syndrom ist mit Adipositas und Großwuchs assoziiert.
C Grenzwertig erhöhte TSH Werte mit hochnormalem fT3 und fT4 findet man bei untergewichtigen Kindern häufiger als bei übergewichtigen Kindern.
D Bei einem erhöhten Kortisolwert im 24-Stunden-Urin sollte man ein Cushing-Syndrom ausschließen.
E Man findet bei den übergewichtigen Kindern häufig eine Hashimoto-Thyreoiditis.

\section{0}

Welche Aussage trifft nicht zu in der Gesprächsführung bei Familien mit einem adipösen Kind?
A Die motivierende Gesprächsführung ist hilfreich.
B Ratschläge sollten durch den Therapeuten entwickelt werden.
C Der Fokus sollte auf Stärken gelegt werden.
D Belohnungssysteme können Verhaltensänderungen bewirken.
E Reframing kann Aussagen der Familien eine neue Deutung geben. 